\title{
CERVICAL MUCUS ARBORIZATION IN THE RHESUS MONKEY
}

\author{
A. DAVID* AND L. MASTROIANNI, JR \\ The Division of Reproductive Biology, \\ Department of Obstetrics and Gynecology, \\ University of Pennsylvania School of Medicine, \\ Philadelphia, Pennsylvania
}

(Received 15th Fanuary 1968)

\begin{abstract}
Summary. Cyclic variations in the quality of cervical mucus were evaluated in the monkey. A characteristic pattern was observed. On the day before ovulation maximal fern production was noted, with a dramatic regression in fern pattern in the subsequent day. A consistent correlation with changes in vaginal cytology was not observed. Daily examination of fern pattern provides a reliable index for ovulation timing in the monkey.
\end{abstract}

\section{INTRODUCTION}

In the field of primate reproduction, the investigator is often handicapped by a lack of availability of an accurate means of ovulation detection. Rectal palpation of the ovaries (Parkes, 1960; Hartman, 1939), vaginal sediments (Corner, 1923) and vaginal smears (Davis \& Hartman, 1935) have been used with varying degrees of success. The present study was designed to evaluate the relationship between ovulation and the status of the cervical mucus in the hope that any cyclic changes would provide yet another parameter for the diagnosis of ovulation in the monkey.

\section{MATERIALS AND METHODS}

Seventeen regularly cycling adult female rhesus monkeys (Macaca mulata) were selected for study. Previously observed menstrual cycles ranged in duration from 27 to 32 days. Daily, beginning at the termination of flow, cervical mucus was collected from the external os by means of a cotton-tipped applicator after exposure of the cervix with a small bivalved speculum. The quantity, viscosity, turbidity and spinnbarkheit were evaluated. The mucus was smeared on a slide, dried over a flame, and examined microscopically for evidence of a 'fern' pattern. In selected animals, a daily vaginal smear was obtained and fixed in $50 \%$ alcohol-ether mixture, stained by Shorr method and evaluated according to the criteria suggested by De Allende, Shorr \& Hartman (1945). In an

* Ford Foundation Postdoctoral Fellow. Permanent address: Tel-Hashomer Hospital, Tel-Aviv Medical School, Tel-Aviv, Israel. 


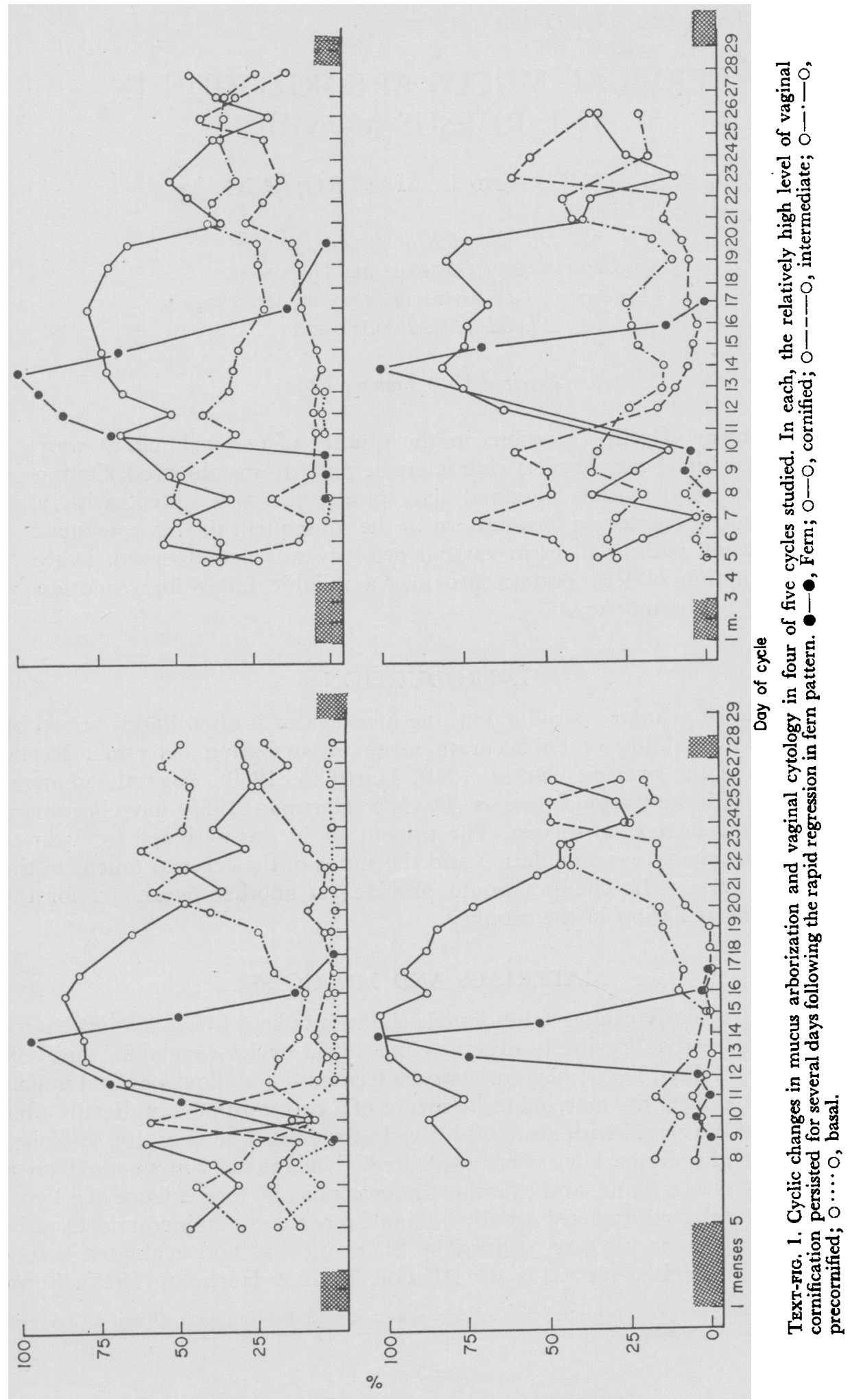


additional seventeen animals, cervical mucus was evaluated immediately before laparotomy, carried out between cycle Days 5 and 28 for ovum and sperm recovery experiments.

\section{RESULTS}

During twenty-five cycles, in which observations were recorded daily, the cervical secretions initially were scanty and displayed high viscosity, no spinnbarkheit and no fern formation. Beginning variously on cycle Days 9 to 16 , a dramatic increase in quantity, decrease in viscosity and some evidence of fern formation were observed. A maximum change was consistently noted 2 days later. At this point, spinnbarkheit was 12 to $15 \mathrm{~cm}$, and fern production was maximal (Plate 1). Thereafter, there was an abrupt regression in fern pattern, and the mucus became viscous once again. Maximum fern production

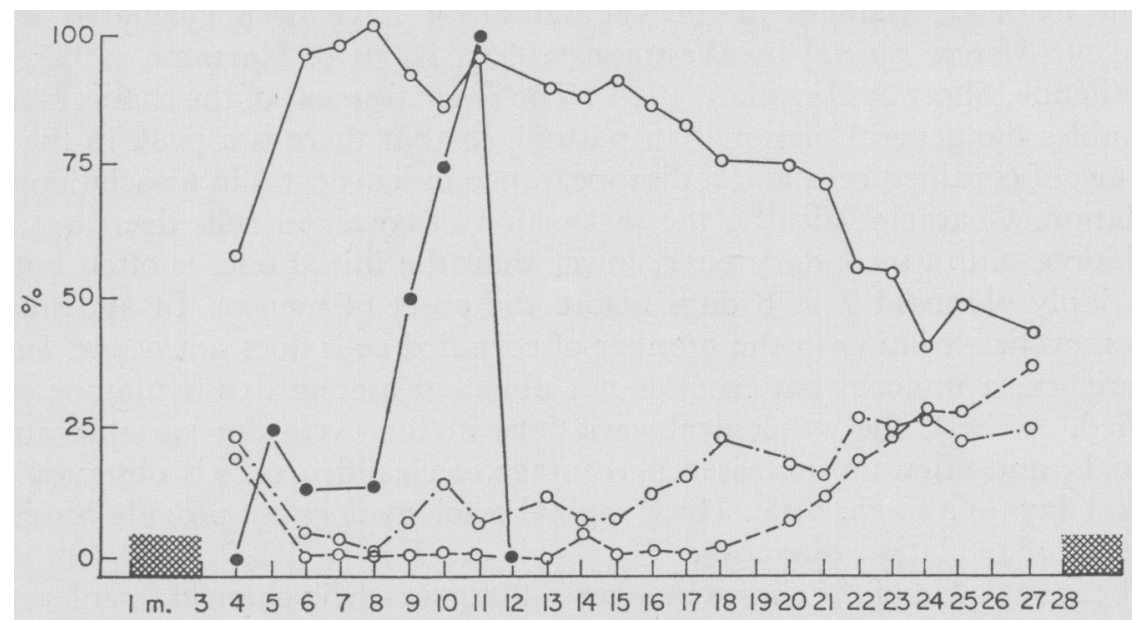

Day of cycle

TEXT-FIG. 2. Cyclic changes in mucus arborization and vaginal cytology in one of five cycles studied in which peak cornification occurred 5 days before the day of maximal

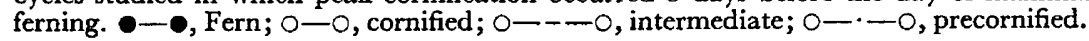

was observed 14 to 15 days before the onset of the next menstrual period in twenty-four cycles. In one, it preceded the onset of flow by 17 days. In five cycles daily vaginal smears were also reviewed. A consistent correlation between maximal fern production and cytologic changes was not observed. In four out of five cycles, however, there was a relatively high percentage of cornified cells on the day of maximum ferning, and the high level of cornification was noted for several days thereafter (Text-fig. 1). In one cycle (Text-fig. 2), peak cornification preceded maximal ferning by several days. There was no consistent cyclic pattern in the percentage of intermediate and precornified cells.

The state of the ovaries was evaluated visually in seventeen animals at various times in the cycle. Maximum fern production was noted on the day of laparotomy in five. In each, the ovaries displayed a fresh punctum, and in 
three a tubal ovum was recovered. In the remainder, the fern pattern was abortive or absent, and the ovaries did not show evidence of recent ovulation. In two, laparotomy was carried out in the presence of an abortive fern, on Days 13 and 14, respectively. Ovulation had not occurred. In these animals, maximal fern patterns were noted on Days 18 and 19, respectively, and in each, menses occurred 14 days later. In three additional animals, laparotomy was performed on Days 14 and 15. There was no precedent fern pattern, and no ovulation points were found. Fern pattern was absent throughout the remainder of the cycle, which terminated on Days 30,31 and 33, respectively. The monkey displaying a 33-day cycle was re-explored on Day 24 and, again, the ovaries did not display gross evidence of corpus luteum formation.

\section{DISCUSSION}

Cyclic cytologic changes in the vaginal smear have been evaluated in the monkey (Macaca mulata) by Westman (1932), Davis \& Hartman (1935) and De Allende, Shorr \& Hartman (1945). The vaginal smear of the rhesus monkey resembles the general mammalian pattern, in that there is a peak in the percentage of cornified cells and a disappearance in leucocytes in association with ovulation. Characteristically, the proportion of cornified cells decreases after ovulation, and a secondary peak, lower than the initial one, is often but not invariably observed 7 to 8 days before the onset of menses. In anovulatory cycles, cyclic variation in the number of cornified cells does not occur. In our experience, a uniform pattern was not observed among five ovulatory cycles studied. At best, the cytological variations in the cycle are variable among animals, and often an increased percentage of cornified cells is observed over several days in a single cycle. Thus, vaginal cytology does not provide consistent criteria for ovulation diagnosis.

The sexual skin of the rhesus monkey undergoes cyclic changes in coloration, most pronounced in the hindquarters and external genitalia (Allen, 1927). In the ovulatory animal, these areas take on a vivid red colour which reaches maximum intensity during the 3rd week after the onset of menses, and fades gradually before the next flow (Zuckerman, 1937). The relationship between these changes and ovulation is not constant, however, and it is generally acknowledged that this index cannot be relied upon for the diagnosis and timing of ovulation.

Rectal bi-manual palpation of ovaries to assess variations in size and consistency has been used for ovulation detection (Hartman, 1939). This method may provide indirect information on ovulation, but interpretation of the findings is difficult.

Changes in the basal body temperature have been observed (Balin, 1966), using sophisticated instruments and telemetry. The cost and complexity of the instrumentation make this approach impracticable.

According to Hamilton (1949), the cells of the endocervix form a single layer, with typical mucous glands. They undergo cyclic variations in height, reflecting the hormonal status of the animal. In anovulatory cycles, epithelial height and secretion are reduced, increasing somewhat during the pre- 
PIATE 1

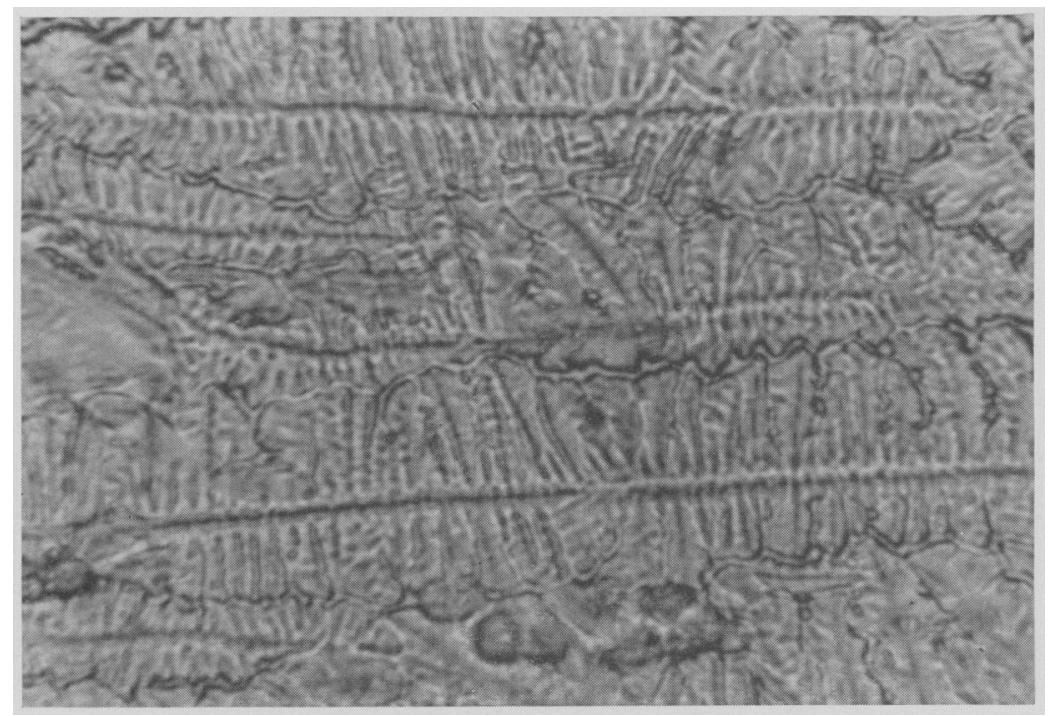

Representative sample of maxinal fern production. $\times 400$.

(Facing p. 498) 
menstrual phase. Hamilton also described cyclic variation in the number of leucocytes in the cervical smear, with a decrease towards mid-cycle.

The cervical mucus is the product of the epithelial cells lining the canal (Stieve, 1927). Under the influence of oestrogens, the height of cells is increased and columns of mucus appear at their free ends, and are discharged into the cervical canal. About the time of ovulation, the physical properties of the mucus change dramatically. The volume increases, and the mucus becomes much less viscous. Similar changes have been observed in the human where the increased volume is a result of accumulation of water (Viergiver \& Pommerenke, 1944); the total solids, notably sodium chloride and reducing sugars, also increase to give the typical 'fern' pattern when dried over a flame (Campas, 1953). The accumulated water and salts modify the physical properties of the mucus. In the macaque monkey, the changes in the cervical mucus display a characteristic pattern. There is maximal fern formation immediately before ovulation, and a rapid regression in fern pattern thereafter. This abrupt change is not observed in the human (David, Insler \& Ber, 1965). In anovulatory cycles, this pattern does not occur. When the follicular phase is prolonged, typical fern pattern appears later in the cycle, but 14 days before onset of menses, suggesting delayed ovulation with a relatively constant luteal phase.

\section{ACKNOWLEDGMENTS}

This work was supported by the Ford Foundation and Public Health Service Grant FR00340.

The authors wish to acknowledge the technical assistance of $\mathrm{Mr}$ Charles Meltzer and Mrs Anne Hoffman.

\section{REFERENCES}

Allen, E. (1927) The menstrual cycle in the monkey Macacus rhesus. Contr. Embryol. 19, 1.

BaLIN, H. (1966) Telemetry and reproductive biology. F.A.A.M.I. 1, 17.

CAmpas, L. (1953) The crystallization test as a guide to the treatment of cervical hostility. Fert. Steril. $4,137$.

Corner, G. W. (1923) Ovulation and menstruation in Macacus rhests. Contr. Embryol. 15, 73.

David, A., Insler, V. \& Ber, R. (1965) The fern test in following treatment with human gonadotrophins. Proc. Tel-Hashom. Hosp. 3, 193.

Davis, M. \& Hartman, C. (1935) Changes in vaginal epithelium during pregnancy in relation to the vaginal cycle. 7. Am. med. Ass. 104, 279.

De Allende, F. L. C., Shorr, E. \& Hartman, C. G. (1945) A comparative study of the vaginal smear cycle of the rhesus monkey and the human. Contr. Embryol. 31, 3.

Hamilton, C. (1949) Observations on the cervical mucosa of the rhesus monkey. Contr. Embryol. 33, 83.

Hartman, G. G. (1939) In: Sex and Internal Secretions, 2nd edn, p. 663. Ed. E. Allen. Williams \& Wilkins, Baltimore.

PARkes, A. S. (1960) Reproductive hormones in birds. In: Marshall's Physiology of Reproduction, 3rd edn, Vol. 1, Pt. 2, p. 621 . Longmans Green, London.

SHORR, E. (1940) A new technic for staining vaginal smears. Science, N. X. 91, 321.

Stieve, H. (1927) Akadem. Verlagsgesellschaft, Leipzig, quoted by C. Hartman (1962) in Science and the safe period. Williams \& Williams, Baltimore.

Viergiver, E. \& Pommerenke, W. (1944) Measurement of the cycle variations in the quantity of cervical mucus and its correlation with basal temperature. Am. J. Obstet. Gynec. 48, 321.

Westman, A. (1932) Acta obstet. gynec. scand. 12, 282 (quoted by A. S. Parkes).

Zuckerman, S. (1937) Menstrual cycle of primates; interaction of ovarian hormones in cycle. Proc. R. Soc. B, 124, 150 . 